\title{
Predictive value of CA 125 and CA 72-4 in ovarian borderline tumors
}

\author{
Miriam S. Lenhard ${ }^{1, *}$, Stefanie Nehring ${ }^{1}$, \\ Dorothea Nagel ${ }^{2}$, Doris Mayr ${ }^{3}$, Angela \\ Kirschenhofer ${ }^{1}$, Linda Hertlein ${ }^{1}$, Klaus Friese ${ }^{1}$, \\ Petra Stieber ${ }^{2}$ and Alexander Burges ${ }^{1}$ \\ ${ }^{1}$ Department of Obstetrics and Gynecology, Ludwig- \\ Maximilians-University Munich, Campus \\ Großhadern, Munich, Germany \\ ${ }^{2}$ Department of Clinical Chemistry, Ludwig- \\ Maximilians-University Munich, Campus \\ Großhadern, Munich, Germany \\ ${ }^{3}$ Department of Pathology, Ludwig-Maximilians- \\ University Munich, Campus Großhadern, Munich, \\ Germany
}

\begin{abstract}
Background: The aim of this study was to assess the prognostic value of cancer antigen (CA) 125 and CA 72-4 in patients with ovarian borderline tumor (BOT). Methods: All women diagnosed and treated for BOT at our institution between 1981 and 2008 were included into this retrospective study $(n=101)$. Preoperatively collected serum samples were analyzed for CA 125 (Architect, Abbott and Elecsys, Roche) and CA 724 (Elecsys, Roche) with reference to clinical data and compared to healthy women $(n=109)$ and ovarian cancer patients $(n=130)$.
\end{abstract}

Results: With a median of $34.7 \mathrm{U} / \mathrm{mL}$ (range 18.1$385.0 \mathrm{U} / \mathrm{mL}$ ) for CA 125 and $2.3 \mathrm{U} / \mathrm{mL}$ (range $0.2-277.0 \mathrm{U} / \mathrm{mL}$ ) for $\mathrm{CA} 72-4$, serum tumor markers in BOT patients were significantly elevated as compared to healthy women with a median CA 125 of 13.5 $\mathrm{U} / \mathrm{mL}$ (range $4.0-49.7 \mathrm{U} / \mathrm{mL}$ ) and median CA 72-4 of $0.8 \mathrm{U} / \mathrm{mL}$ (range $0.2-20.6 \mathrm{U} / \mathrm{mL}$ ). In addition, there was a significant difference compared with ovarian cancer patients who showed a median CA 125 of $401.5 \mathrm{U} / \mathrm{mL}$ (range $12.5-35,813 \mathrm{U} / \mathrm{mL}$ ), but no difference was observed for CA 72-4 (median $3.9 \mathrm{U} / \mathrm{mL}$, range $0.3-10,068 \mathrm{U} / \mathrm{mL}$ ). Patients with a pT1a tumor stage had significantly lower values of CA 125 but not of CA 72-4 compared with individuals with higher tumor stages (median CA $12529.9 \mathrm{U} / \mathrm{mL}$ for pT1a vs. $50.9 \mathrm{U} / \mathrm{mL}$ for $>\mathrm{pT} 1 \mathrm{a} ; \mathrm{p}=0.014)$. There was a trend for increased concentrations of CA 125 but not of CA 72-4 in the presence of ascites, endometriosis or peritoneal implants at primary diagnosis. With respect to the prognostic value of CA 125 or CA 72-4, CA 125

*Corresponding author: Miriam Lenhard, MD, Department of Obstetrics and Gynecology, Ludwig-MaximiliansUniversity Munich, Campus Großhadern,

Marchioninistraße 15, 80337 Munich, Germany Phone: + 49-89-7095-0, Fax: + 49-89-7095-6724,

E-mail: Miriam.Lenhard@med.uni-muenchen.de

Received December 18, 2008; accepted March 2, 2009; previously published online March 25, 2009 was significantly higher at primary diagnosis in patients who later developed recurrence $(251.0 \mathrm{U} / \mathrm{mL}$ vs. $34.65 \mathrm{U} / \mathrm{mL}, \mathrm{p}=0.012$ ).

Conclusions: Serum CA 125 and CA 72-4 concentrations in BOT patients differ from healthy controls and patients with ovarian cancer. CA 125, but not CA 724 , at primary diagnosis correlates with tumor stage and tends to be increased in the presence of ascites, endometriosis or peritoneal implants. Moreover, CA 125 at primary diagnosis appears to have prognostic value for recurrence.

Clin Chem Lab Med 2009;47:537-42.

Keywords: biomarker; CA 125; CA 72-4; ovarian borderline tumor (BOT); prognosis; tumor marker.

\section{Introduction}

Ovarian borderline tumors (BOT) account for $10 \%-20 \%$ of epithelial ovarian tumors (1). Often described as a tumor of low malignant potential, it is classified as a separate entity by the International Federation of Gynecology and Obstetrics (2). It has an incidence of 4.8/100,000 per year (3), and an excellent clinical outcome reflected by 10 -year survival rates of $\sim 90 \%$ (4). Recurrence rates according to the literature vary between $8 \%$ and $32 \%(5-9)$. Few studies have analyzed risk factors for disease recurrence or prognosis (9-11). Since borderline tumors of the ovary have been described as showing late recurrence (12), long-term follow-up is required to assess prognosis.

There is little information on the prognostic value of preoperative tumor markers in BOT. It is known that preoperative cancer antigen (CA) 125 concentrations are increased in fewer patients with BOT compared to ovarian cancer (13). In ovarian cancer, the magnitude of increase in CA 125 is related to tumor stage and histological subtype (14). In early ovarian cancer, CA 125 concentrations at initial diagnosis have been shown to be of prognostic value for survival (15). The aim of this study was to assess the prognostic value of preoperative CA 125 and CA 72-4 in patients diagnosed with BOT.

\section{Materials and methods}

\section{Patients}

Study group I Since different methods for measurement of CA 125 had been used over the long-time period that we evaluated, two different study groups were established. Study group I comprised healthy women, BOT and ovarian cancer patients, and was used to validate the measurement of tumor markers using different testing systems. 
In addition, the concentration of tumor markers in BOT patients, patients with ovarian cancer and healthy women were compared in this patient group. In total, this group consisted of 16 patients diagnosed with a BOT, 130 patients with ovarian cancer and 109 healthy women for whom blood serum samples were available. Statistical evaluation of this group assessed tumor marker concentrations, patient age, menopause, tumor stage and histology.

Study group II All women diagnosed and treated for BOT between 1981 and 2008 at our institution were included in the retrospective analysis. A total of 160 patients treated for BOT were identified. Of these 160 patients, tumor marker concentrations had been measured preoperatively at our institution in 101 patients, who were assigned to study group II. For the remaining 59 patients, preoperative tumor marker measurements were either not performed or had been done outside our institution. Therefore, these patients were excluded to ensure standardized analysis and comparability of results. In the retrospective evaluation, tumor marker concentrations were evaluated with respect to patient characteristics and clinical information including histology, tumor stage, endometriosis, ascites, menopausal status, relapse and survival.

Tumor markers Serum samples from patients included in study group I had been stored at $-80^{\circ} \mathrm{C}$. CA $72-4$ (Elecsys, Roche Diagnostics, Penzberg, Germany) and CA 125 (Architect, Abbott Diagnostics, Chicago, US and Elecsys, Roche Diagnostics, Penzberg, Germany) were measured in patients diagnosed with BOT $(n=16)$, ovarian cancer $(n=130)$ and healthy women $(n=109)$. CA 125 and CA 72-4 concentrations for study group II had been measured at the time of primary diagnosis using the kit in use at that time.

Histology All patients underwent surgery by experienced gynecological surgeons at our institution. Typing and staging of tumors were performed by the Department of Pathology according to the criteria of the International Federation of Gynaecologists and Obstetricians (FIGO) and the International Union against Cancer (IUCC). Tumor, nodes, metastases (TNM) and FIGO stage, World Health Organization (WHO) grading and the presence of peritoneal implants were recorded.

Follow-up Clinical data, demographic, diagnostic and treatment information were obtained primarily from the patients' charts. The following parameters were recorded for each patient at initial diagnosis: age, menopausal status, and history of endometriosis.

During follow-up, patients were seen every 3 months following initial diagnosis for a period of three years, then at six-month intervals for an additional two years, and then once a year. Patients were evaluated sonographically for signs of relapse and tumor markers were measured at each follow-up visit. If a relapse was suspected (e.g., a persisting suspicious adnexal mass found at sonography or a continuous rise in tumor markers) surgery was performed to achieve a histological diagnosis. The occurrence of relapse, time to relapse, death and survival time were recorded. Disease recurrence and survival were the primary outcomes assessed.

Statistics Statistical analysis was performed using SAS V9.1 (SAS Institute Inc., Cary, NC, USA). Tumor marker concentrations are expressed as median and range. Differences between subgroups of group I and II were evaluated using the Wilcoxon test. For comparison of relapse and survival times in group II, Kaplan-Meier curves were constructed. Two groups were differentiated using median tumor marker values as cut-off points, and the $\chi^{2}$ statistic of the log-rank test was calculated to test for differences between survival curves. For regression analysis, the PHREG procedure was performed based on the Cox proportional hazard model. Tumor marker values were transformed to the logarithm base 2 scale as predictors. p-Values $<0.05$ were considered statistically significant.

\section{Results}

\section{Study group I}

Median age of patients at diagnosis of BOT was 59.6 years (range $23.6-88.3$ years), for ovarian cancer 62.8 years (range $22.7-88.2$ years). These reflect representative ages for diagnosis of an ovarian borderline or ovarian cancer. Median age for the group of healthy women was 38.4 years (range 21.5-80.0 years). Due to the higher median age in the ovarian cancer patients and BOT group, there was a higher rate of postmenopausal women $181.3 \%$ in BOT, $78.5 \%$ in ovarian cancer patients vs. $19.2 \%$ in healthy women). Histological stage was determined to be stage I in $15(93.8 \%)$ and stage III in one $(6.2 \%)$ of the BOT patients. For patients diagnosed with ovarian cancer, $22(16.9 \%)$ had stage I disease, stage II in 15 (11.5\%), stage III in $83(63.8 \%)$ and stage IV in 10 patients (7.7\%) (Table 1).

All samples were tested for CA 125 using two different test systems (Architect, Abbott and Elecsys, Roche). Significantly different CA 125 concentrations were observed between patients with a diagnosis of ovarian cancer, BOT, and healthy women. Results for BOT showed a median value of $34.7 \mathrm{U} / \mathrm{mL}$ (range 18.1-385 U/mL) measured with the Architect and $36.6 \mathrm{U} / \mathrm{mL}$ (range 17.3-332 U/mL) using the Elecsys system. Results for CA 125 in BOT patients, obtained using either system, were comparable and not statistically different $(p>0.05)$. However, for cancer patients, median CA 125 was $401.5 \mathrm{U} / \mathrm{mL}$ (range $12.5-35,813 \mathrm{U} / \mathrm{mL}$ ) with the Architect and $391.5 \mathrm{U} / \mathrm{mL}$ (range 13-27,070 U/mL) with the Elecsys system $(p<0.001)$ (Table 1). Healthy women had a median CA 125 of $13.5 \mathrm{U} / \mathrm{mL}$ (range $4.0-49.7 \mathrm{U} / \mathrm{mL}$ ) using the Architect and $11.9 \mathrm{U} / \mathrm{mL}$ (range 3.2-68.8 U/mL) using the Elecsys system $(p<0.001)$. Interestingly, for both tumor markers none of the patients diagnosed with BOT or ovarian cancer had CA 125 levels below 10 $\mathrm{U} / \mathrm{mL}$, while more than $50 \%$ of healthy women showed CA 125 levels below $10 \mathrm{U} / \mathrm{mL}$.

Healthy women had significantly lower CA 72-4, median $0.8 \mathrm{U} / \mathrm{mL}$ (range 0.2-20.6 U/mL), compared to BOT and ovarian cancer patients $(p=0.03)$ (Table 1). There was no significant difference between CA 72-4 concentrations in patients with BOT (median 2.3 $\mathrm{U} / \mathrm{mL})$ and ovarian cancer $(3.9 \mathrm{U} / \mathrm{mL})(\mathrm{p}=0.29)$.

Comparison of tumor marker concentrations measured in the same samples from individuals in group I using the different test systems showed no significant differences. Thus, the different testing systems used for tumor marker measurement in group II over the 
Table 1 Patient characteristics of study group I comparing patients with BOT (borderline ovarian tumor), Ov CA (ovarian cancer) and healthy women.

\begin{tabular}{llll}
\hline Patient characteristics & BOT & Ov CA & Healthy women \\
\hline $\mathrm{n}$ & 16 & 130 & 109 \\
Age (median), years & 59.6 & 62.8 & 38.3 \\
Premenopausal, \% & 18.7 & 21.5 & 78.5 \\
Postmenopausal, \% & 81.3 & 22 & \\
Stage I, n & 15 & 15 & \\
Stage II, n & 0 & 83 & \\
Stage III, n & 1 & 10.8 \\
Stage IV, n & 0 & $401.5(12.5-35,813)$ \\
CA 125 U/mL (median, range) & & $391.5(13-27,070)$ \\
$\quad$ Architect, Abbott & $34.7(18.1-385.0)$ & & $13.5(4.0-49.7)$ \\
$\quad$ Elecsys, Roche & $36.6(17.3-332.0)$ & $11.9(3.2-68.8)$ \\
CA 72-4 U/mL (median, range) & & $3.9(0.3-10068)$ \\
$\quad$ Elecsys, Roche & $2.3(0.2-277)$ & $0.8(0.2-20.6)$ \\
\hline
\end{tabular}

years that this study encompassed should not have any effect on the retrospective data evaluation of group II study patients.

\section{Study group II}

Tumor marker measurements and surgery was performed on 101 patients with BOT. Mean (SD) followup time was 6.3 (4.6) years (minimum three months, maximum 19.9 years). Relapse occurred during the follow-up period in five patients $(4.95 \%)$ with a mean time to recurrence of $2.5(16.0)$ years (minimum three months, maximum 4.6 years). Five deaths $(4.95 \%)$ occurred, although it was not known if these were tumor-related. Median age at primary diagnosis was 53 years (range $18-88$ years) and $61.5 \%$ of patients were premenopausal. Histology revealed a serous tumor in $63 \%$ of patients, mucinous in $36 \%$, and endometrioid in one patient. Table 2 shows that $62.2 \%$ of all patients were diagnosed at an early tumor stage (pT1a).

Increased tumor marker concentrations were found in $51.5 \%$ of BOT patients. Patients with increased CA

Table 2 Patient characteristics of study group II.

\begin{tabular}{ll}
\hline Patients, $\mathrm{n}$ & 101 \\
Age (median), years & 53 years (range 18-88) \\
Menopausal stage, \% & \\
$\quad$ Premenopausal & 61.5 \\
$\quad$ Postmenopausal & 38.5 \\
Histology, \% & \\
$\quad$ Serous & 63.0 \\
$\quad$ Mucinous & 36.3 \\
$\quad$ Endometrioid & 1.0 \\
Tumor stage, \% & \\
pT1a & 62.2 \\
>pT1a & 37.8 \\
Ascites, \% & 10.8 \\
Peritoneal implants, \% & \\
$\quad$ All & 9.9 \\
Invasive & 8.9 \\
Endometriosis, \% & 9.9 \\
Follow-up (median) years & $6.3 \pm 4.6$ years \\
$\quad$ & (minimum 3 months, \\
$\quad$ Relapse, \% & maximum 19.9 years) \\
Deaths, \% & 5.0 \\
\hline
\end{tabular}

125 and CA 72-4 did not show any statistical difference with regard to menopausal status or histological subtype $(p>0.05)$ (Table 3). Patients with tumor stage pT1a had significantly lower values for CA 125 but not for CA 72-4 compared to those with higher tumor stages (CA 125: $29.9 \mathrm{U} / \mathrm{mL}$ for pT1a vs. $50.9 \mathrm{U} / \mathrm{mL}$ for higher stages; $p=0.014 ; C A$ 72-4: $1.7 \mathrm{U} / \mathrm{mL}$ vs. 2.3 $\mathrm{U} / \mathrm{mL} ; \mathrm{p}=0.165)$.

There was a trend for increased concentrations of CA 125, but not of CA 72-4, in the presence of ascites (CA 125, $p=0.289$; $C A$ 72-4, $p=0.686$ ) endometriosis (CA 125, $p=0.116$; CA 72-4, $p=0.697$ ) or peritoneal implants (CA 125, $\mathrm{p}=0.057$; CA 72-4, $\mathrm{p}=0.928$ ) at primary diagnosis.

The prognostic value of CA 125 was significantly higher at initial diagnosis in patients who later developed recurrent disease $(251.0 \mathrm{U} / \mathrm{mL}$ vs. $34.65 \mathrm{U} / \mathrm{mL}$, $p=0.012$ ). Similar findings were also observed for CA $72-4(p=0.093)$. Kaplan-Meier curves for CA 125 and CA 72-4 revealed a significant relationship between relapse and elevated CA 125 (Log-rank test, CA 125: cut-off $35 \mathrm{U} / \mathrm{mL}, p=0.0368$; CA 72-4: cut-off $1.9 \mathrm{U} / \mathrm{mL}$, $p=0.0976$ ) (Figures 1 and 2). Cox regression model analysis showed the doubling of CA 125 to be associated with a 2.261-fold risk of disease recurrence (CA 125: $p=0.0039$, hazard ratio (HR) $2.26,95 \% \mathrm{HR}$ confidence interval (CI) 1.30-3.93; CA 72-4: $p=0.1977$, HR $1.27,95 \% \mathrm{HR} \mathrm{Cl} 0.88-1.82$ ). However, no statistically significant findings were found for overall survival.

\section{Discussion}

Information on the association of preoperative tumor marker findings and BOT is very limited $(16,17)$. Studies of CA 125 have shown increased tumor marker concentrations in BOT as well as in patients with ovarian cancer (18). Our data confirm that serum CA 125 and CA 72-4 in patients with BOT differ in median values from healthy controls and patients with ovarian cancer. The latter group generally shows the highest concentration of CA 125 and CA 72-4; lowest concentrations seen in healthy women. Data from the literature suggest that the prevalence of BOT patients with increased tumor marker concentrations varies between $15 \%$ and $50 \%$ (19). These data are further 
Table 3 CA 125 and CA 72-4 concentrations according to menopausal stage, histology, tumor stage, presence of ascites, peritoneal implants and endometriosis.

\begin{tabular}{|c|c|c|c|c|}
\hline \multirow{2}{*}{$\overline{\text { Menopausal status }}$} & \multicolumn{2}{|c|}{ CA 125 U/mL (median, range) } & \multicolumn{2}{|c|}{ CA $72-4 \mathrm{U} / \mathrm{mL}$ (median, range) } \\
\hline & & & & \\
\hline Premenopausal & $36.9(2.6-1996)$ & & $1.95(0.4-396)$ & \\
\hline Postmenopausal & $29.5(7.3-418)$ & $p=0.668$ & $2.0(0.2-396)$ & $p=0.406$ \\
\hline \multicolumn{5}{|l|}{ Histology } \\
\hline Serous & $35.2(2.6-1996)$ & & $1.7(0.2-396)$ & \\
\hline Mucinous & $36.1(7.0-418)$ & $p=0.647$ & $2.3(0.2-277)$ & $p=0.243$ \\
\hline Endometrioid & 427.0 & & 42.0 & \\
\hline \multicolumn{5}{|l|}{ Tumor stage } \\
\hline pT1a & $29.9(2.6-454)$ & & $1.7(0.2-230)$ & \\
\hline$>\mathrm{pT} 1 \mathrm{a}$ & $50.9(7.3-1996)$ & $\mathrm{p}<0.01^{\mathrm{a}}$ & $2.3(0.2-396)$ & $p=0.165$ \\
\hline \multicolumn{5}{|l|}{ Ascites } \\
\hline Yes & $62.8(2.6-1996)$ & & $2.2(0.5-396)$ & \\
\hline No & $35.2(7.0-418)$ & $p=0.029$ & $1.9(0.2-277)$ & $p=0.687$ \\
\hline \multicolumn{5}{|l|}{ Implantation } \\
\hline Yes & $122.5(7.3-1996)$ & & $2.2(0.2-396)$ & \\
\hline No & $35.3(2.6-529)$ & $p=0.06$ & $1.85(0.4-277)$ & $p=0.929$ \\
\hline \multicolumn{5}{|l|}{ Endometriosis } \\
\hline Yes & $98.6(17.6-418)$ & & $1.7(0.5-7.2)$ & \\
\hline No & $35.3(2.6-1996)$ & $p=0.12$ & $2.0(0.2-396)$ & $p=0.697$ \\
\hline
\end{tabular}

aStatistically significant.

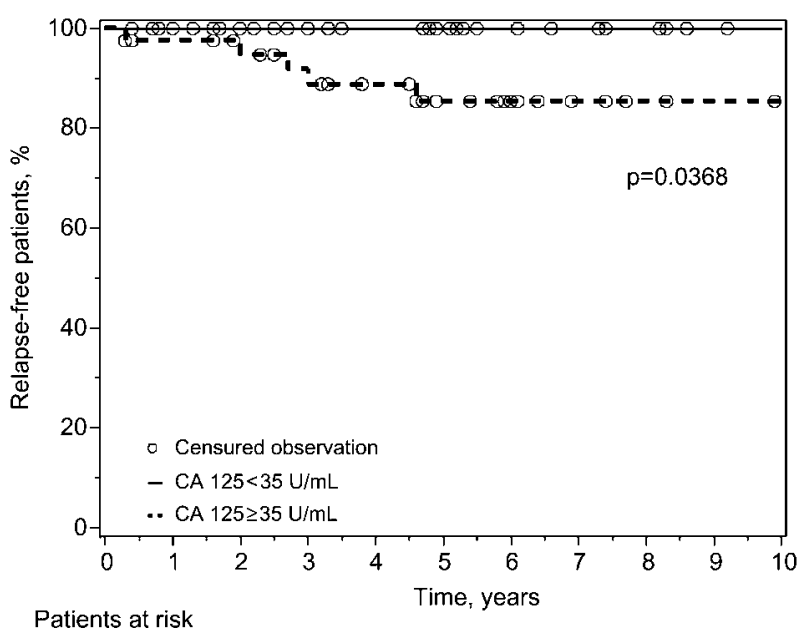

\begin{tabular}{|r|r|r|r|r|r|r|r|r|r|r|}
\hline$<35$ & 42 & 35 & 31 & 26 & 24 & 21 & 17 & 14 & 12 & 8 \\
\hline$\geq 35$ & 42 & 39 & 36 & 32 & 29 & 23 & 19 & 13 & 11 & 10 \\
\hline
\end{tabular}

Figure 1 Relapse-free patients with reference to CA 125.

supported by our study which showed tumor marker increases in $51.5 \%$ of BOT patients. It has been previously reported that CA 125 is often not released in patients with mucinous tumors (20), though our data showed comparable results for mucinous and serous tumors.

To date, the diagnosis of a BOT cannot be made solely with CA 125 and CA 72-4 measurements because of the considerable variability and overlap between patients with ovarian cancer and healthy individuals. We found that CA 125 , but not CA 72-4, correlates with tumor stage at initial diagnosis. Also CA 125 tends to be increased in the presence of ascites, endometriosis or peritoneal implants. Data from the literature suggest that $80 \%-85 \%$ of patients with advanced stages of ovarian cancer show increased concentrations of CA 125 , while only $50 \%$ with stage I disease show such increases $(14,21)$.
Our findings may differ if results were interpreted with respect to normal baseline values rather than cut-off thresholds. Normally, baseline CA 125 and CA 72-4 measurements are not performed in healthy women, making it difficult to test this hypothesis. However, there are data showing that CA 125 levels rise over time in patients with BOT, before the tumor becomes clinically evident and is diagnosed (18). Since we know that CA 125 and CA 72-4 concentrations are increased in patients with BOT, knowledge of a persistent increase in tumor marker concentrations in these women might be helpful in establishing a diagnosis. In addition, we have shown that CA 125 can be useful as a good negative predictor since all patients diagnosed with BOT or ovarian cancer had CA 125 levels above $10 \mathrm{U} / \mathrm{mL}$, but more than $50 \%$ of healthy women showed CA 125 levels below $10 \mathrm{U} / \mathrm{mL}$. This may be especially useful when confronted with

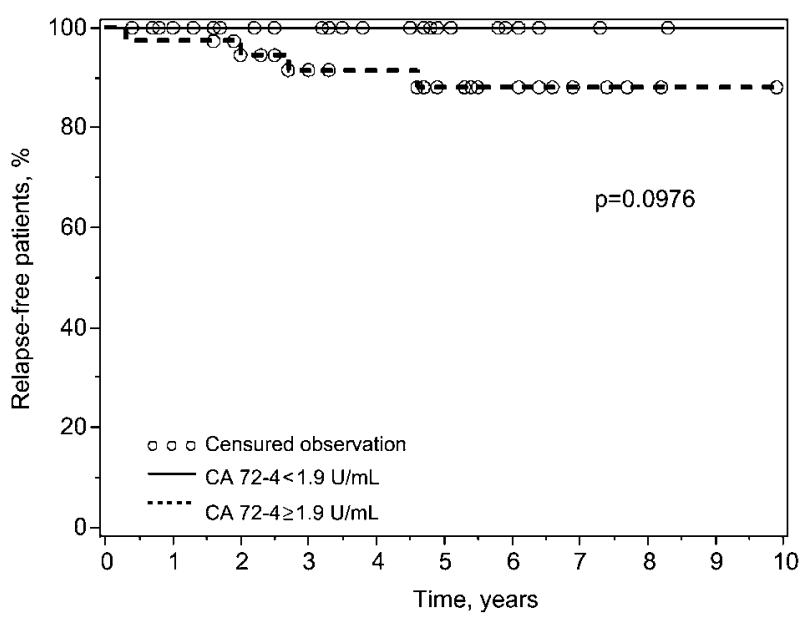

Patients at risk

\begin{tabular}{|r|r|r|r|r|r|r|r|r|r|r|}
\hline$<1.9$ & 32 & 25 & 22 & 20 & 16 & 11 & 8 & 5 & 4 & 3 \\
\hline$\geq 1.9$ & 37 & 36 & 34 & 28 & 27 & 23 & 20 & 14 & 11 & 9 \\
\hline
\end{tabular}

Figure 2 Relapse-free patients with reference to CA 72-4. 
unclear sonographic imaging findings where CA 125 can be useful for identifying patients at low-risk for BOT or ovarian cancer.

The interpretation of increased tumor marker concentrations is still difficult because it is known to be influenced by inflammation, endometriosis, liver disease, and ovulation, and also differs in pre- and postmenopausal women $(13,22)$. The high rate of false-positive results make interpretation of CA 125 and CA 72-4, alone, difficult in gynecological patients (22). Therefore, combination with sonography and clinical exam remains essential for diagnosis and decision making.

Although, patients diagnosed with BOT generally have a good prognosis, recurrence can occur. The rate of recurrence in BOT is rather low, with a relapse rate of $5 \%$ in this study group, compared to published data showing rates of $15 \%-20 \%$ (23). At present, the identification of patients at risk for recurrence of BOT remains uncertain. Morice et al. found patients with invasive implants to have higher relapse rates (11). Our data show CA 125 elevation at initial diagnosis to be of prognostic value for recurrence. Therefore, CA 125 and CA 72-4 in addition to being helpful in the diagnosis of BOT, might even be useful for the identification of patients at risk for disease recurrence by allowing appropriate follow-up intervals to be established, since even late recurrence has been described (12).

The role of tumor markers for early detection of disease recurrence during clinical follow-up remains difficult. Rustin et al. found that an increase in serum CA 125 to more than twice the upper limit of normal during follow-up in patients with ovarian cancer after first line chemotherapy accurately predicts tumor relapse (24). Gotlieb et al., studying patients with BOT, showed that increased concentrations of CA 125 can indicate relapse, although increases did not occur in all patients with relapse (16). Zanetta et al. described increases in CA 125 above the cut-off in only $17 \%$ of patients at the time of relapse (25). This finding is in agreement with our data, as we also observed that not all patients with recurrent disease showed an increase in CA 125. However, it is not clear whether early diagnosis of recurrent disease is of prognostic advantage. Since better data are not available, clinical examination, ultrasound and tumor marker measurement remain the basis for clinical follow-up of patients with BOT.

The strength of this study is the long-term followup of patients (27 years), the high standard of surgery by gynecologic oncologists at a specialized academic institution and histopathologic review by expert gynecologic oncology pathologists. A limitation is the retrospective study design.

\section{Conclusions}

Serum CA 125 and 72-4 in patients with BOT differ in median values from healthy controls and ovarian cancer patients. CA 125 , but not CA 72-4, correlates with tumor stage at initial diagnosis and tends to be increased in the presence of ascites, endometriosis or peritoneal implants. In addition, CA 125 concentrations at initial diagnosis appear to have prognostic value for disease recurrence. However, better analytic tools for the detection of BOT are desirable.

\section{References}

1. Barakat RR. Borderline tumors of the ovary. Obstet Gynecol Clin North Am 1994;21:93-105.

2. Scully RE. World Health Organization classification and nomenclature of ovarian cancer. Natl Cancer Inst Monogr 1975;42:5-7.

3. Trope C, Kaern J. Management of borderline tumors of the ovary: state of the art. Semin Oncol 1998;25:372-80.

4. Trimble CL, Trimble EL. Management of epithelial ovarian tumors of low malignant potential. Gynecol Oncol 1994;55:S52-61.

5. Kennedy AW, Hart WR. Ovarian papillary serous tumors of low malignant potential (serous borderline tumors). A long-term follow-up study, including patients with microinvasion, lymph node metastasis, and transformation to invasive serous carcinoma. Cancer 1996; 78:278-86.

6. Crispens MA. Borderline ovarian tumours: a review of the recent literature. Curr Opin Obstet Gynecol 2003;15: 39-43.

7. Lackman F, Carey MS, Kirk ME, McLachlin CM, Elit L. Surgery as sole treatment for serous borderline tumors of the ovary with non-invasive implants. Gynecol Oncol 2003;90:407-12.

8. Prat J, De Nictolis M. Serous borderline tumors of the ovary: a long-term follow-up study of 137 cases, including 18 with a micropapillary pattern and 20 with microinvasion. Am J Surg Pathol 2002;26:1111-28.

9. Seidman JD, Kurman RJ. Ovarian serous borderline tumors: a critical review of the literature with emphasis on prognostic indicators. Hum Pathol 2000;31:539-57.

10. Morice P, Camatte S, Rouzier R, Pautier P, Atallah D, Pomel $C$, et al. [Prognostic factors and treatment for advanced-stage borderline ovarian tumors]. J Gynecol Obstet Biol Reprod (Paris) 2002;31:623-8.

11. Morice P, Camatte S, Rey A, Atallah D, Lhomme C, Pautier $P$, et al. Prognostic factors for patients with advanced stage serous borderline tumours of the ovary. Ann Oncol 2003;14:592-8.

12. Silva EG, Gershenson DM, Malpica A, Deavers M. The recurrence and the overall survival rates of ovarian serous borderline neoplasms with non-invasive implants is time dependent. Am J Surg Pathol 2006;30:1367-71.

13. Kenemans P, Yedema CA, Bon GG, von Mensdorff-Pouilly S. CA 125 in gynecological pathology - a review. Eur J Obstet Gynecol Reprod Biol 1993;49:115-24.

14. Young RC, Walton LA, Ellenberg SS, Homesley HD, Wilbanks GD, Decker DG, et al. Adjuvant therapy in stage I and stage II epithelial ovarian cancer. Results of two prospective randomized trials. N Engl J Med 1990;322: 1021-7.

15. Paramasivam S, Tripcony L, Crandon A, Quinn M, Hammond I, Marsden D, et al. Prognostic importance of preoperative CA-125 in International Federation of Gynecology and Obstetrics stage I epithelial ovarian cancer: an Australian multicenter study. J Clin Oncol 2005; 23:5938-42.

16. Gotlieb WH, Soriano D, Achiron R, Zalel Y, Davidson B, 
Kopolovic J, et al. CA 125 measurement and ultrasonography in borderline tumors of the ovary. Am J Obstet Gynecol 2000;183:541-6.

17. van Nagell JR Jr, DePriest PD, Ueland FR, DeSimone CP, Cooper AL, McDonald JM, et al. Ovarian cancer screening with annual transvaginal sonography: findings of 25,000 women screened. Cancer 2007;109:1887-96.

18. Zurawski VR Jr, Orjaseter H, Andersen A, Jellum E. Elevated serum CA 125 levels prior to diagnosis of ovarian neoplasia: relevance for early detection of ovarian cancer. Int J Cancer 1988;42:677-80.

19. Buy JN, Ghossain MA, Sciot C, Bazot M, Guinet C, Prevot $S$, et al. Epithelial tumors of the ovary: CT findings and correlation with US. Radiology 1991;178:811-8.

20. Zurawski VR Jr, Knapp RC, Einhorn N, Kenemans P, Mortel $\mathrm{R}$, Ohmi $\mathrm{K}$, et al. An initial analysis of preoperative serum CA 125 levels in patients with early stage ovarian carcinoma. Gynecol Oncol 1988;30:7-14.

21. Duffy MJ, Bonfrer JM, Kulpa J, Rustin GJ, Soletormos G, Torre GC, et al. CA125 in ovarian cancer: European group on tumor markers guidelines for clinical use. Int J Gynecol Cancer 2005;15:679-91.

22. Medeiros LR, Rosa DD, da Rosa MI, Bozzetti MC. Accuracy of CA 125 in the diagnosis of ovarian tumors: a quantitative systematic review. Eur J Obstet Gynecol Reprod Biol 2009;142:99-105.

23. Zanetta G, Rota S, Chiari S, Bonazzi C, Bratina G, Mangioni C. Behavior of borderline tumors with particular interest to persistence, recurrence, and progression to invasive carcinoma: a prospective study. J Clin Oncol 2001;19:2658-64.

24. Rustin GJ, Nelstrop AE, Tuxen MK, Lambert HE. Defining progression of ovarian carcinoma during follow-up according to CA 125: a North Thames Ovary Group Study. Ann Oncol 1996;7:361-4.

25. Zanetta G, Rota S, Lissoni A, Meni A, Brancatelli G, Buda A. Ultrasound, physical examination, and CA 125 measurement for the detection of recurrence after conservative surgery for early borderline ovarian tumors. Gynecol Oncol 2001;81:63-6. 